\title{
Multimodality image fusion for diagnosing coronary artery disease
}

\author{
Marina Piccinelli ${ }^{\square}$, Ernest Garcia \\ Department of Radiology and Imaging Sciences, Emory University, Atlanta 30322, GA, USA. \\ Received 04 September 2013, Accepted 16 September 2013, Epub 28 September 2013
}

\begin{abstract}
Coronary artery disease (CAD) is one of the leading causes of death in the US and a substantial health-care burden in all industrialized societies. In recent years we have witnessed a constant strive towards the development and the clinical application of novel or improved detection methods as well as therapies. Particularly, noninvasive imaging is a decisive component in the cardiovascular field. Image fusion is the ability of combining into a single integrated display the anatomical as well as the physiological data retrieved by separated modalities. Clinical evidence suggests that it represents a promising strategy in CAD assessment and risk stratification by significantly improving the diagnostic power of each modality independently considered and of the traditional side-by-side interpretation. Numerous techniques and approaches taken from the image registration field have been implemented and validated in the context of CAD assessment and management. Although its diagnostic power is widely accepted, additional technical developments are still needed to become a routinely used clinical tool.
\end{abstract}

Keywords: image fusion, CAD diagnosis, computed tomography angiography, nuclear imaging

\section{INTRODUCTION}

Cardiovascular diseases (CVD) are the leading cause of death in most Western societies. Statistics from recent updates from the American Heart Association $^{[1,2]}$ report that an estimated 82.6 millions of American adults, i.e. $>1$ in 3 , have one or more types of CVD. Coronary artery disease (CAD) is estimated to cause alone about 1 every five deaths and results in an estimated total annual cost of US \$165.4 billion. Although its mortality rate has decreased in the last decade, CAD prevalence is projected to increase over the next 20 years remaining a significant healthcare burden for all industrialized and developing countries $^{[3]}$.

\footnotetext{
$\square$ Corresponding author: Marina Piccinelli, Ph.D., Department of Radiology and Imaging Sciences, Emory University, Room 1203, 101 Woodruff Circle, Atlanta, GA 30322, USA. Tel/Fax: +1-404-727-
}

As a result of the profound economical and social impact of CAD prevalence, in conjunction with the persistence of many of its risk factors (e.g. obesity, diabetes, hypertension, sedentary life), in recent years we have witnessed a constant strive towards the development and the clinical application of novel or improved detection methods as well as therapies. Particularly, noninvasive imaging is a decisive component in the cardiovascular field, affecting all stages of disease management: from a correct and possibly early diagnosis, to guidance through available therapeutic solutions, to the selection of the patients that would benefit from those choices.

Today, most common noninvasive imaging modalities to investigate CAD include myocardial perfusion

6113/+1-404-727-3488, E-mail: mpiccin@emory.edu.

The authors reported no conflict of interests. 
imaging (MPI), performed by means of single photon emission computed tomography (SPECT) or positron emission tomography (PET), and computed tomography angiography (CTA). All modalities have their own weaknesses and strengths and which test will be initially performed on a particular patient depends mostly on the symptoms and the pretest likelihood of CAD. CTA is primarily considered an anatomical study to image the coronary tree that supplies blood to the myocardium, potentially capable of replacing invasive coronary angiography (CA); SPECT and PET are highly reliable physiological tests conveying information about myocardial perfusion (blood flow) and viability. A substantial amount of literature indicates that a comprehensive technique for CAD assessment should provide the complementary information about coronary morphology and myocardial function. The concept of 'one-stop-shop' -i.e. one imaging modality that could alone assess all aspects of ischemic disease has intrigued clinicians for some time ${ }^{[4]}$, but despite that technical improvements are continuously being implemented, each technique will likely keep bringing its own specific advantages, at least in the cardiac field.

Instead, both cardiologist and researchers in the field agree that image fusion, i.e. the possibility of combining into a single integrated display the clinical data independently retrieved by each modality, represents a new promising strategy in cardiovascular diagnosis and risk stratification. The integration is achieved by way of software methods globally catalogued under the term of image registration techniques that align two different types of images of the cardiovascular system obtained either sequentially and, to some extent, simultaneously, into a single framework. In its most successful cardiac application, image fusion overlays anatomical and functional datasets: for example, CTA and SPECT may be combined, so that each hypoperfused region can be directly linked to a specific stenosis along the coronary tree. In the fused image the two studies work synergistically increasing not only the diagnostic power of each modality, but also of their more traditional side-by-side interpretation $^{[5]}$.

In the following sections the more widely used noninvasive imaging modalities for CAD assessment will be presented, and advantages and disadvantages of each approach will be outlined putting forward the rationale for multimodality image fusion. A general description of the image registration framework and its essential technical requirements will be introduced. Main techniques and results of image fusion and its clinical potential will be reported. This review will conclude underlying the existing limitations of image fusion approaches in the reality of clinical settings and patient well-being.

\section{NON-INVASIVE IMAGING MODALI- TIES FOR CAD ASSESSMENT}

\section{Computed tomography angiography}

Although the gold standard for the characterization of coronary morphology remains invasive CA, contrast-enhanced, electrocardiographically gated CTA is the most promising non-invasive modality to visualize both calcified and non-calcified plaques and to estimate the degree of stenosis with a minimum prerequisite of 64-multidetector row device. Given the tremendous advances in terms of temporal and spatial resolution, CTA has been given significant attention in recent years; a number of single center and multicenter studies have been published detailing its capability in correctly diagnosing CAD with respect to $\mathrm{CA}^{[6,7,8,9]}$. In Fig. 1, a normal case and a case with left anterior descending artery disease are depicted by means of CA, reformatted CTA and CTA-derived volume rendering technique.

The main advantage of CTA is the excellent negative predictive value (NPV) - that is the ability of ruling out the presence of disease - in symptomatic patients with low pre-test likelihood of CAD, but even in situations of acute chest pain ${ }^{[10-13]}$. The main disadvantage is the suboptimal positive predictive value (PPV) - that is the ability to correctly diagnose the presence of disease - for both $>50 \%$ and $>70 \%$ stenoses. The low PPV is due to the frequent overestimation of luminal narrowing especially in situation of densely calcified plaques. Calcifications induce blooming artifacts in the images, which in turn prevent the correct and reliable assessment of the degree of stenosis; most commonly this results in overestimation of the severity of the disease or in technically inadequate studies that prevent the accurate assessment of certain vascular segments. Additionally, since CTA cannot evaluate ischemic burden, even if obstructive disease is detected the functional relevance of a measured stenosis cannot be estimated ${ }^{[14-17]}$ and consequently the actual need for revascularization assessed. This is particularly true for intermediate lesions for which the questions on whether CA would be needed and catheterization would be effective ${ }^{[18]}$ may remain unanswered on the basis of the CTA test alone. There are situations where CTA alone could answer the clinical question. Two examples are patients with normal coronaries and high-risk patients with CTA-based diagnosis of 2- or 3-vessel coronary 

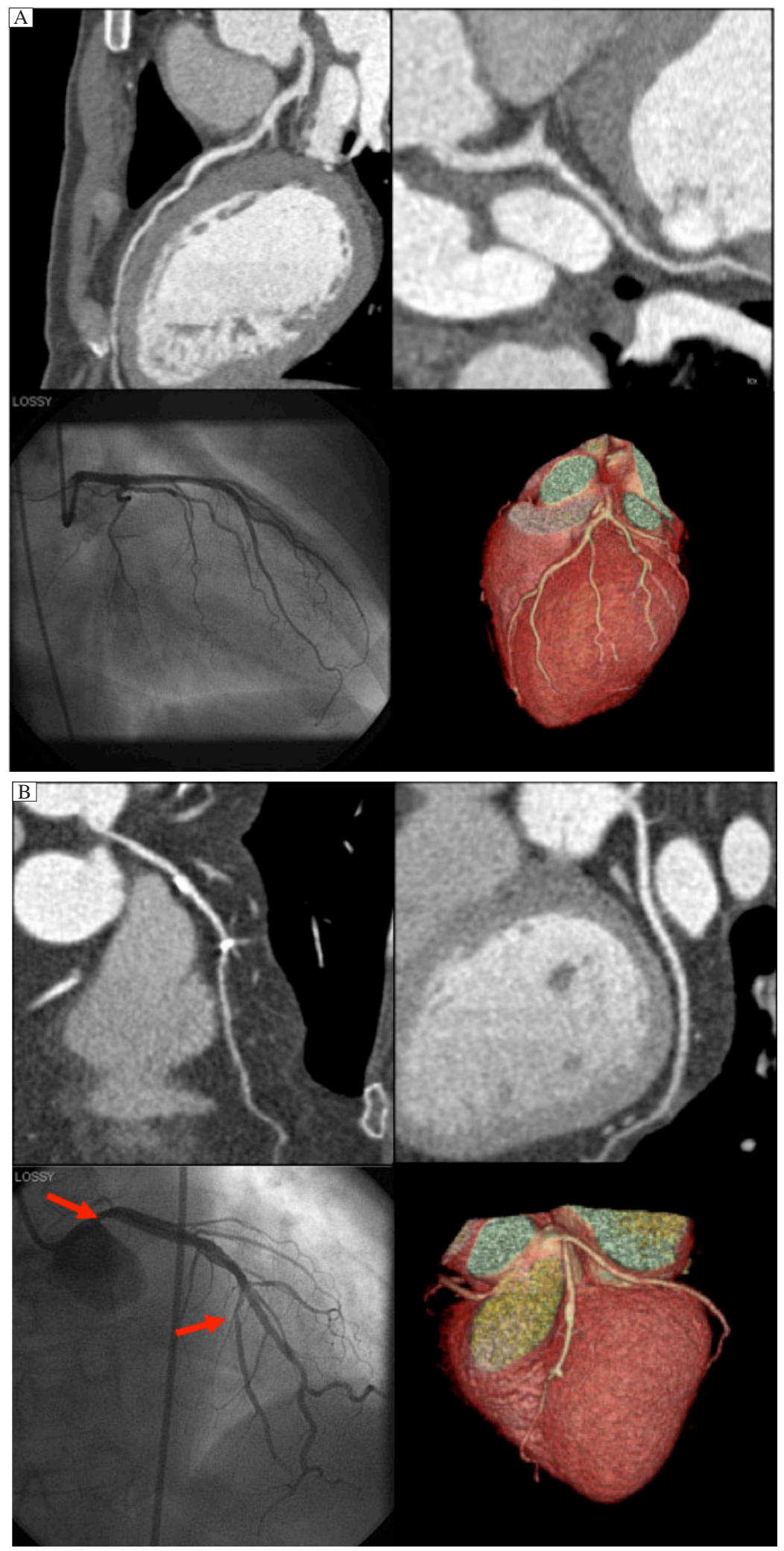

Fig. 1 A normal case (A) and a case with LAD disease (B) visualized by means of reformatted CTA (top), CA views (bottom, left) and CTA-derived volume rendering technique (bottom, right). Red arrows identify significant stenosis $>50 \%$. Images courtesy of Dr C. Santana.

disease and involvement of the proximal left anterior descending (LAD) coronary artery will most likely benefit from revascularization ${ }^{[19]}$.
Apart from the aforementioned limitations in evaluating densely calcified plaques and intermediate lesions, CTA necessitates the administration of an 
intravenous iodinated contrast, which is particularly contraindicated in patients with renal dysfunction. In patients with high heart rate, poor quality images may also result from difficulties in performing gated acquisitions. The radiation exposure is also a limiting factor to the widespread use of CTA, although recent developments and new protocols allow acquisitions with low radiation dose ${ }^{[20]}$.

\section{Myocardial perfusion imaging: SPECT and PET}

Rest and stress myocardial perfusion imaging, utilizing SPECT and PET radionuclide techniques, are today the most efficient noninvasive methodology for the detection and quantification of obstructive CAD. Presence of reversible perfusion defects of tracer accumulation are interpreted as a sign of exercise-induced hypo-perfusion, while fixed defects at rest and stress as proxies for myocardial scar ${ }^{[21,22]}$. The strength of nuclear cardiology resides in the extensive amount of clinical and scientific publications supporting the use of ischemia measurements in guiding patient management and therapeutical decisions. Addition- ally, in the last decade, a number of software packages have been developed for the fully automated and objective quantification of myocardial perfusion images and extraction of clinical data. These automated software packages have allowed the standardization and widespread utilization of nuclear cardiology as the most reliable diagnostic test for CAD disease evaluation and patient risk stratification ${ }^{[23,24,25]}$. In Fig. 2, the active view ECTb display of a patient is shown ${ }^{[23]}$. the quantitative analysis includes: planar projections, oblique slices, polar maps including normal database comparison, 3D displays, 17-segment summed score polar maps, LV volume/time curve and regional thickening map.

\section{SPECT}

Single photon emission computed tomography is the most frequently performed MPI technique in the US for CAD assessment. Mean sensitivity and specificity for the detection of $>50 \%$ stenosis are, respectively, $86 \%$ and $74 \%{ }^{[26]}$. Robust data support its prognostic value ${ }^{[27-30]}$ : the basic assumption is that risk of adverse events is directly linked to the size of the

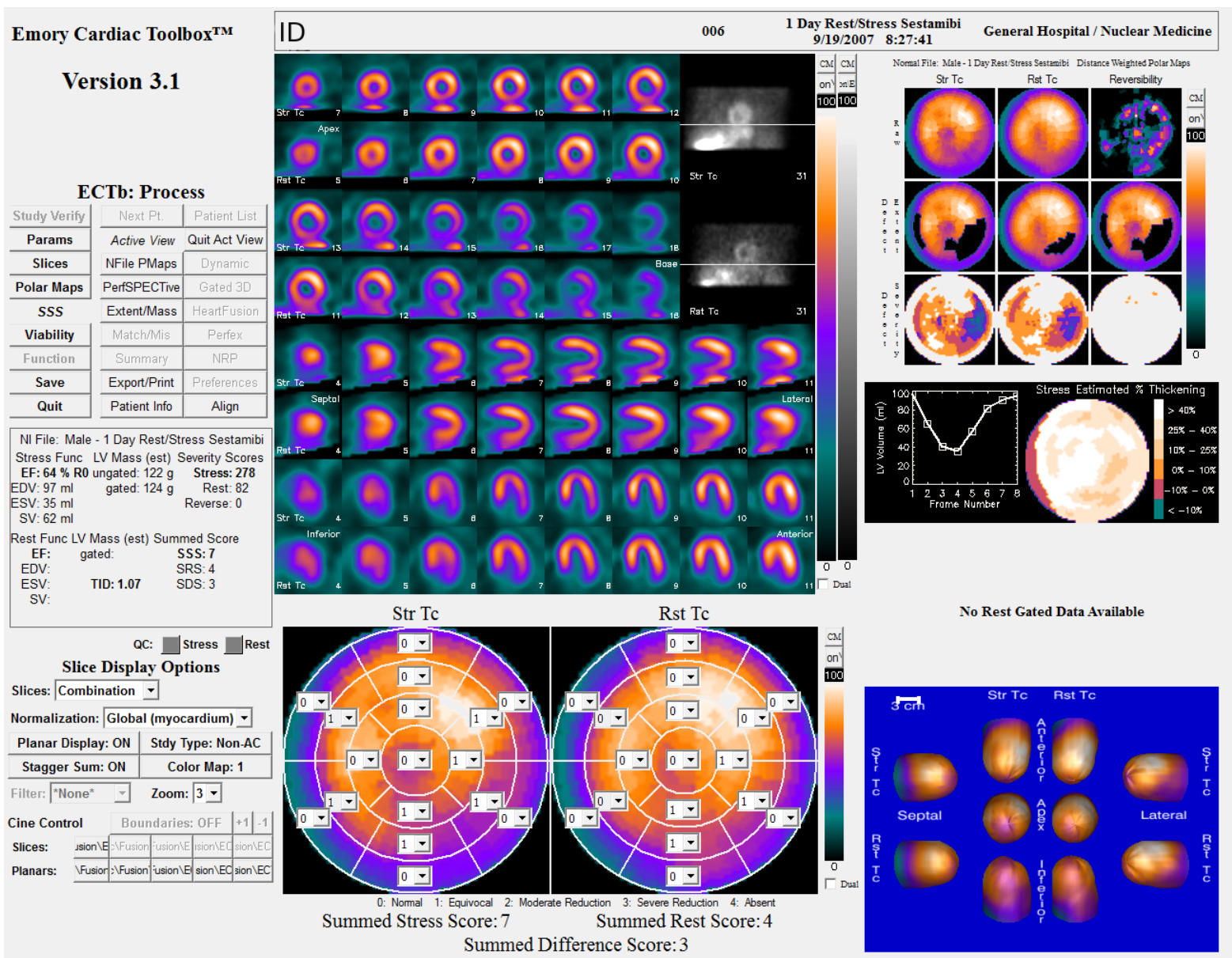

Fig. 2 Active view display ECTb23. The display integrates different types of image quantification: planar projections, oblique slices, polar maps including normal database comparison, 3D displays, 17-segment summed score polar maps, LV volume/time curve and regional thickening map. 
perfusion defect, which in turn reflects the extent of obstructive CAD; the larger the defect, the worse the prognosis. Annual rate of events for patients with normal stress scans is $0.8 \%$ while it increases to annually $5.6 \%$ for abnormal scan.

The main disadvantages of SPECT imaging are that attenuation artifacts can be interpreted as perfusion defects and that SPECT commonly underestimates CAD extent in case of multi-vessel disease and LAD involvement, particularly in patients with diabetes. The latter is due to a mechanism called "balanced ischemia" that reduces contrast heterogeneity between normally and abnormally perfused territories, affecting the test ability to distinguish between them. MPI tests reveal ischemia in areas supplied by the most severely stenosed vessels and no detection of subclinical atherosclerosis is possible. This is indeed expected since MPI is designed to extract functional information, i.e. flow-limiting stenoses.

A crucial drawback when dealing with MPI interpretation is the lack of anatomical information. Myocardial distribution territories are traditionally assigned to their subtending arteries by the use of the 17-segment model as proposed by the American Heart Association ${ }^{[31]}$; it is, however, documented that in $17 \%$ to $50 \%$ of patients the standard modeling does not correspond to the actual coronary anatomy ${ }^{[32]}$, further complicating the identification of the stenosis responsible for a specific perfusion defect.

\section{PET}

Positron emission tomography (PET) shares many aspects with SPECT in terms of pros and cons. It can be considered as an alternative test although until recently its diffusion as a standard clinical study was hampered by the limited availability of devices and the complex production of proper radiopharmaceuticals. With respect to SPECT, PET is characterized by a higher spatial and temporal resolution and benefits from depth-independent attenuation correction techniques, which makes it the gold standard of myocardial perfusion imaging. Its sensitivity and specificity are slightly better $\left(90 \%\right.$ and $89 \%$, respectively ${ }^{[9]}$ ), as it is the capability of detecting patients with multi-vessel disease. As opposed to SPECT, PET allows the accurate quantitative measurement of absolute myocardial blood flow at rest and stress (measured in $\mathrm{mL} / \mathrm{g} / \mathrm{min}$ ); the coronary flow reserve can further be computed as the ratio between myocardial blood flow at stress and the blood flow at rest. The clinical use of these measures has been shown to have strong additional prognostic power in risk stratification especially in difficult cases with multiple vessel disease $\mathrm{e}^{[33-35]}$.

\section{Image fusion: more than the sum}

The routine application of CTA and MPI for the assessment of CAD provide complementary diagnostic information for a comprehensive picture of disease severity. Another attribute of combining the use of these two techniques is that their weaknesses affect different aspects of the complex task of CAD detection and management. Whenever one of the two approaches, anatomical or functional, fails to be decisive in detecting disease or guiding clinical decisions, the other one can complement the diagnosis with additional information and help solving borderline findings. Densely calcified vessels will not affect image quality in SPECT or PET studies; multi-vessel disease or left main coronary artery stenosis, which is often misread with MPI, is, on the other hand, well diagnosed by means of CTA. Intermediate lesions can be difficult to classify on CTA unless functional formation on the subtending myocardial perfusion is provided, while even in the presence of a defect, MPI scans may not clearly identify the vessels responsible for the hypo-perfusion, unless patient-specific coronary tree anatomy is given.

The excellent ability of this noninvasive integrated information to guide CAD management and limit the use of CA to the patients that will truly benefit from revascularization techniques was demonstrated in a number of studies ${ }^{[36,37]}$. Patients will benefit from invasive procedures only when flow-limiting stenoses are present and ischemia proven; on the opposite, aggressive treatment of non-flow-limiting stenoses - which is not without periprocedural risks - will not significantly change patient's prognosis or symptoms ${ }^{[38]}$. In their work, Gaemperli and co-workers reported that by CTA-MPI integration all flow-limiting stenoses were identified, but also that a consistent number of cases that did not show flow-limiting stenoses or myocardial ischemia eventually underwent unnecessary revascularization. The authors concluded that non-invasive combined imaging approach could prevent unnecessary procedures and guide patient selection.

Thus, image fusion does not just deal with the convenience of having an additional cardiovascular test in ambiguous cases (it is estimated that $15-30 \%$ of patients will require multimodality imaging tests ${ }^{[38]}$ ), but, with the actual merging in the same visualization, display the two types of information simultaneously. Traditionally, multimodal imaging studies have been viewed and analyzed separately in a side-by-side fashion and mentally combined together to clear doubts or inconsistencies. In a study specifically designed to address this issue ${ }^{[5]}$, the authors examined the incre- 
mental diagnostic value provided by fusion of SPECT and CTA studies with respect to MPI evaluation alone and CTA-MPI side-by-side approach. After the initial diagnosis with only the MPI scans physicians were presented with the CTA datasets, which allowed them to modify their reports in $50 \%$ of the cases; when the fused displays were provided, reviewers changed their decisions in $28 \%$ of the cases. The confidence in interpretation was higher with software-integrated images with decreased number of misinterpretations and better accuracy, particularly in the LAD territory (Fig. 3).

Analogously, Gaemperli and coworkers ${ }^{[39]}$ demonstrated that in one third of their cardiac patients the fused CTA-SPECT analysis provided additional diagnostic information with respect to the side-by-side interpretation of stand-alone CTA and SPECT acquisitions. Particularly, cases with lesions in the distal and diagonal segments benefited by the integrated display. The ability of fused images to correctly establish the hemodynamic significance of an intermediate lesion on CTA was further underlined as it may help avoiding unnecessary interventional procedures.

\section{MEDICAL IMAGE REGISTRATION, THEORY AND METHODS}

Image fusion is the procedure of integrating relevant information from two or more images into a single one so that the result will be more informative than any of the input singularly considered. From the technical point of view, image fusion applies techniques from the more general field of image registration $^{[40,41,42]}$. The final goal is the spatial alignment of two images so that anatomical or functional features can be directly co-localized in both datasets point-bypoint. The increasing use of radiological images obtained with different modalities and at different points in time has made image registration a very active field in medical image processing. Particularly, automated registration techniques have been the focus of considerable investigation ${ }^{[43,44]}$. Interactive alignment is still an option in research applications, often used as reference for validation purposes, but lacks reproducibility and can be critically time-consuming when translated to a clinical setting.

Three main components have to be defined for a registration procedure to be correctly outlined: a transformation model, a similarity metric and an optimization method. The process of image registration will find the optimal geometric transformation that maximizes the correspondences across the two images, the floating image - the one that has to be registered - and the reference image. All three com- ponents are application-dependent and a number of choices exist.

The transformation model defines how the coordinates between the images are related. The rigid body transformation is the most common choice: it assumes that the distance between any two points in the body remains the same irrespective of the image modality. The only possible geometric transformations between the two representations are rotations and translations along $\mathrm{X}, \mathrm{Y}$ and $\mathrm{Z}$ for a total number of six parameters - scaling may also be included adding three more parameters. Eventually, a single coordinate transformation maps each position in the floating image to the anatomically correspondent point in the reference one. Non-rigid transformations are employed when the hypothesis of rigidity cannot be applied, which typically happens when breathing motions or tissue movements between the two acquisitions cannot be disregarded. In this case, a global mapping between floating and reference image cannot be written; instead a rigid registration is commonly used as a starting estimate and the non-rigid component is modeled as a deformation field that represents the displacement vector required to align the datasets for each point of the image lattice. More physical models have also been broadly investigated to mathematically characterize how tissues may move; common examples are fluid ${ }^{[45]}$, diffusion ${ }^{[46]}$ and elastic models ${ }^{[47]}$. The complexity of non-rigid transformations with respect to the rigid ones is greatly increased from both the theoretical and computation point of view, but rapid progresses have been made in the field and many applications benefit from the higher accuracy granted by these types of models ${ }^{[48]}$.

The similarity metric quantifies the degree of alignment between two given images; during the registration process, which usually employs iterative algorithms, the similarity measure increases until a maximum is reached. A large variety of metrics have been proposed, mostly because the quantification of "matching" depends on the type of information that will be used to actually align the data. Two main approaches are available: feature-based and voxelbased. In the feature-based approach a pre-processing step is required to extract from the images anatomic landmarks or geometric characteristics, such as lines or edges or organ contours, and the similarity measure quantifies the "distance" between corresponding landmarks or surfaces ${ }^{[49]}$. Among the most used numerical techniques are the iterative closest point (ICP) algorithm for matching clouds of points ${ }^{[50]}$ and the "head and hat" method. The head and hat method was specifically applied to intermodality registration of a high-resolution anatomical dataset with a low- 

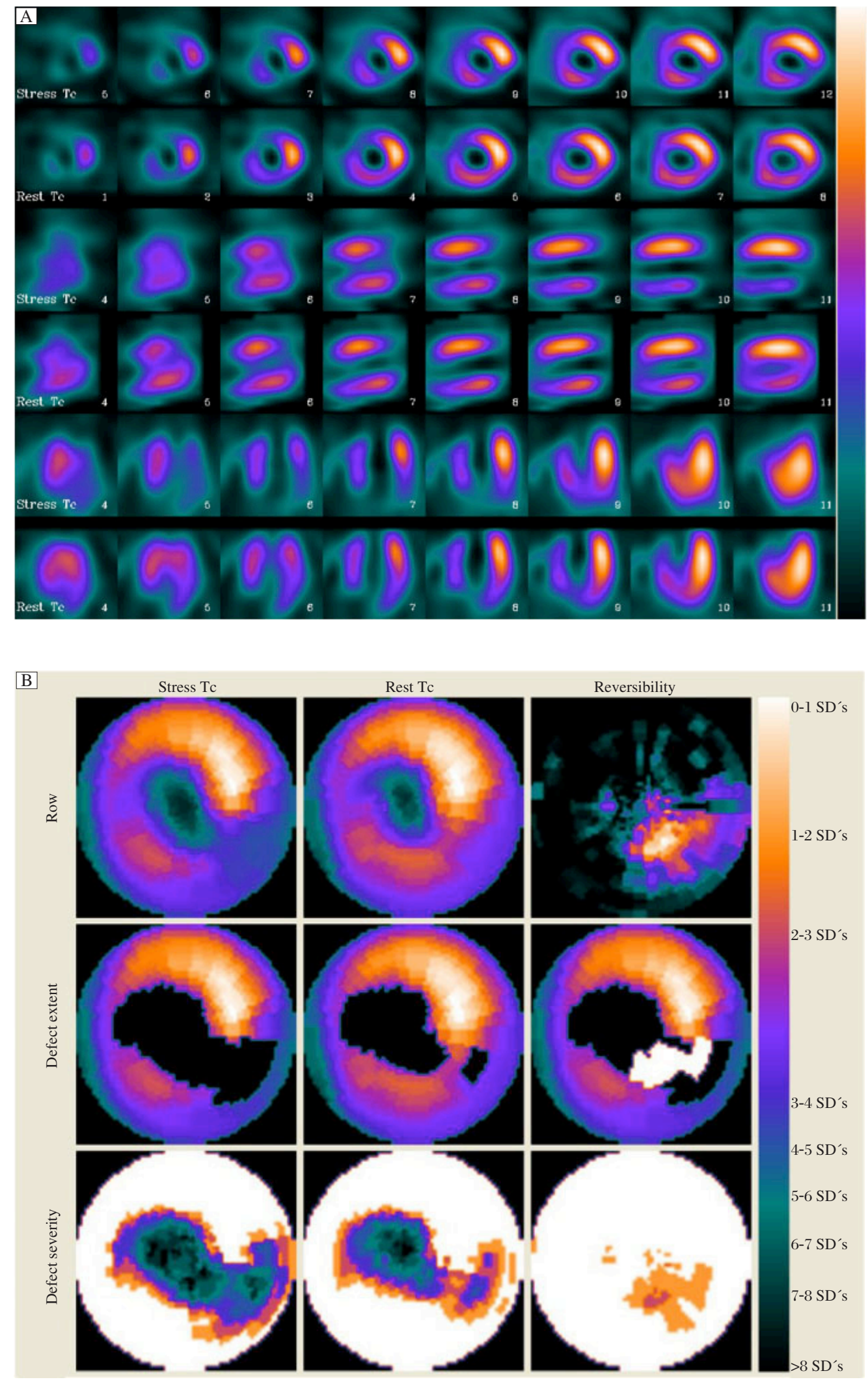

Fig. 3 Example of fusion display versus side-by-side interpretation for a patient with multi-vessel coronary artery disease. A: Short-axis, vertical and horizontal long-axis slices of stress/rest SPECT. B: corresponding polar maps. 

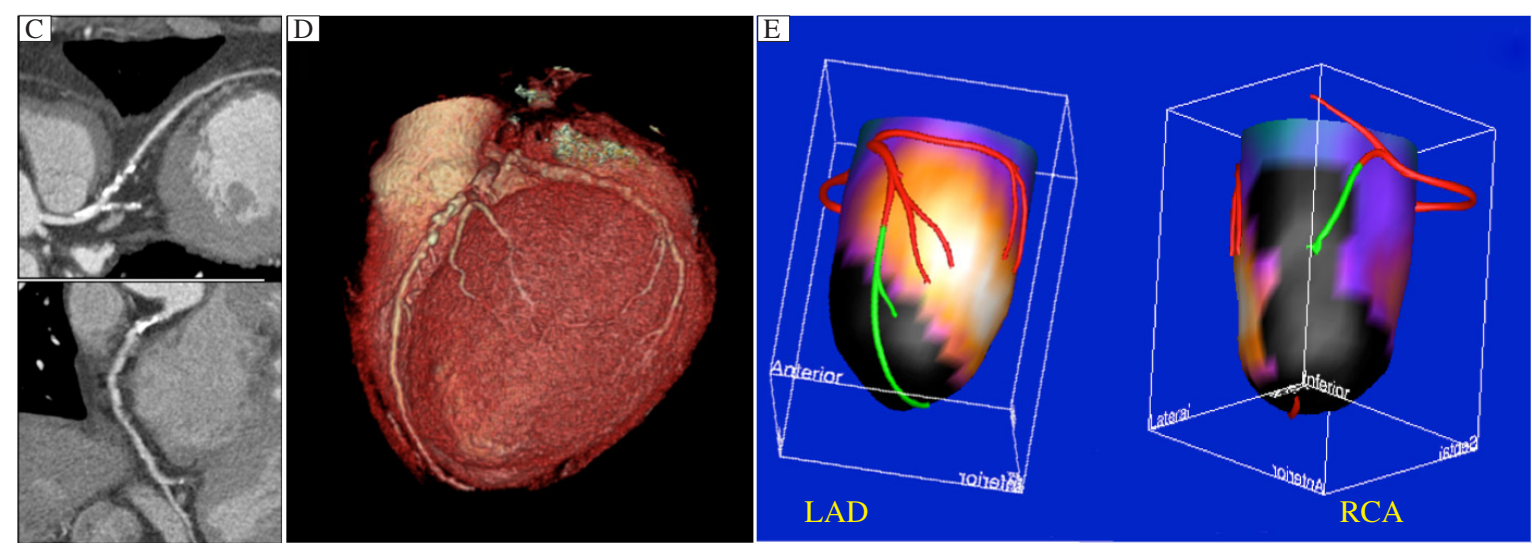

Fig. 3 Example of fusion display versus side-by-side interpretation for a patient with multi-vessel coronary artery disease (continued). C: CTA multiplanar reformatted images of LAD (top) and RCA (bottom). D: volume-rendered display; E: two views of the fused display obtained by means of the algorithm described i ${ }^{[66]}$. The black area on the integrated image identifies the regions of myocardial hypoperfusion during stress. The tracts of the coronary tree in green color are segments distal to the stenoses. The fused display was read as positive for coronary artery disease and confirmed by invasive coronary angiography. Images courtesy of Dr C. Santana.

resolution functional one ${ }^{[51]}$, as it commonly occurs in image fusion for CAD assessment ${ }^{[52,53]}$. The great advantage of feature-based approach is its suitability for both intra and intermodality registration, but requires a preliminary segmentation step tailored to each modality and high accuracy to avoid segmentation errors to adversely affect the registration results. Voxel-based approaches directly use the intensity values from the two datasets: in case of intramodality registration, they directly compare the datasets voxel by voxel, or compute the sum of intensity differences $\left(\mathrm{SSD}^{[45]}\right)$ or the normalized cross correlation $\left(\mathrm{NCC}^{[45]}\right)$. For the more recurrent multimodality registration, techniques from the information theory framework have been applied: image registration should in fact align images so that the content of shared information is maximized in the combined image. To this task, metrics to quantify information in a given image have been used, such as Shannon-Wiener entropy and the joint entropy measures $^{[54]}$, mutual information ${ }^{[55]}$ (MI) and normalized mutual information ${ }^{[56]}$. Methods maximizing MI have shown the most promising results ${ }^{[57,58]}$ : MI is a measure of how well one image can "explain" the other in terms of the information it contains; no assumption is made on image intensities, which makes it particularly effective in multimodality imaging.

Once the transformation model and the similarity metric have been defined, an optimization method must be selected. An optimization method is numerical routine that iteratively searches the space of the transformation parameters, evaluates the agreement between the transformed image and the reference one at that particular step and stops when some predefined level of matching has been reached. The computa- tional burden can become easily cumbersome depending on the size of the images, the number of the transformation parameters and the method selected. Many different algorithms are available ranging in speed and reliability: Powell's method, simplex algorithm, gradient descent method and least squares approaches ${ }^{[59]}$. To improve both speed and accuracy of the selected algorithm, the coarse-to-fine approach is often selected, where instead of the original images, coarser ones are initially used to obtain an optimal transform; then the resolution is progressively refined and the previously identified transform used as an initialization for the new registration process.

\section{IMAGE FUSION IMPEMENTATIONS}

Over the last decade, many studies and research groups have tried, tested and validated different image fusion procedures in the context of clinical CAD assessment and management.

The initial attempts to implement image fusion and test its potential appear in the works of Pfeifer et al. ${ }^{[60]}$ and Faber et al. ${ }^{[6]]}$ and they coincide with the ability of reconstructing 3D models of the coronary trees and of the LV epicardial surface from respectively biplane $\mathrm{CA}$ and perfusion studies. Given that the two models are semi-automatically obtained ${ }^{[62,63]}$, Faber and colleagues describe a technique to unify them based on an optimization approach. A cost function consisting in the distance of the LAD to the interventricular groove and the left circumflex artery (LCX) to the atrioventricular groove is minimized using a conjugate gradient technique. The method was validated in an animal study and showed an excellent spatial correspondence between stenosed artery and abnormally perfused ar- 
eas. Schindler and coworkers ${ }^{[64]}$ designed a methodology to retrieve the 3D coronary tree from two angiographic views specifically selected to allow optimal visualization of the vessels and minimization of foreshortening. The vessels were manually segmented by following their course on the images, placing fiduciary points and interpolating the final paths with Bezier curves. After an initial manual masking to remove extra cardiac activity, automatic quantification of MPISPECT was performed to obtain a triangulated surface of the left ventricular (LV) epicardium; the surface was color-coded with the perfusion information. The fusion procedure was performed in three successive steps the angiographic data were transformed to the MPI coordinate system; information about the calibration of the angiographic images allowed the coronary tree to be scaled to match the size of the ventricular surface; a final 3D translation matched the coronary tree to the left ventricle surface. The authors determined that in $74 \%$ of the cases the stenosis manually identified by an expert user on the CA corresponded to perfusion defects. In a more recent paper, Faber et al. ${ }^{[65]}$ analogously extracted the coronary tree anatomy from CA and registered it to MPI-SPECT. The nuclear image analysis was automatically performed by means of the ECTb ${ }^{[23]}$, a software package for objective and reliable quantification of MPI images that allow the extraction, among many other features, of the LV epicardial surfaces. The angiographic images were manually segmented with an ad hoc software tool to extract the coronaries centerline; 3D models of the coronary trees were eventually created with either constant radius or by applying edge-detection techniques on the original images once the centerlines have been delineated. The fusion process envisaged three phases: an initial rough alignment of the coronary tree with the SPECT image, followed by a more refined one and a final non-linear warping of the coronary models to adapt to the LV surface. The registration was performed by initially aligning the left anterior descending artery (LAD) and the posterior descending artery (PDA) with the anterior and posterior interventricular grooves, respectively ${ }^{[6]}$, and successively applying the ICP algorithm to further improve the alignment. In the final step, the distance between each coronary point and the LV surface was computed and the coronary points translated by the same distance onto the surface. As a validation of the complete procedure, two areas were computed on the LV surface: one from the quantification of the perfusion abnormalities - the myocardial 'blacked-out' area - and one to estimate the myocardial area at risk due to the presence of the blockage; good agreement in the overlap of these areas confirmed the accuracy of the registration procedure. In Fig. 3-E, an example of the fused display obtained with this procedure is shown. This possibility of performing a quantitative image fusion can be an additional asset to the physicians' understanding of difficult cases and their confidence in making therapeutical decisions as compared to more qualitative approaches that mostly rely on efficient visualization techniques of both datasets ${ }^{[67]}$.

The previous examples are mostly application of feature-based registration. In this approach, the original images are pre-processed to segment objects or identify anatomical landmarks that are in turn used to align the datasets. Although the coronary segmentation may require some adjustments, the general approach may be applied to non-invasive CTA acquisitions with relatively small changes.

A voxel-based approach was instead developed by Slomka et al. ${ }^{[68]}$ for the automated registration of CTA and SPECT images. Pre-processing of the original images was still performed: coronary tree anatomy was extracted using vendors' software tools in a semiautomated fashion; LV myocardium and blood pool were automatically identified in MPI images and new volumetric dataset with masked myocardium and blood pool was created; the CTA image was smoothed with a Gaussian filter to remove noise. The whole images - CTA and preprocessed MPI - both oriented along the transverse axis, were used as inputs to the registration framework. The rigid transformation was applied, the SSD was used as a similarity metric and gradient descent as optimization method. To avoid unreasonable solutions and speed the process the coarse-to-fine approach was implemented. The final optimal transformation was eventually used to register the coronary tree on the MPI surfaces.

In all these implementations, rigid transformations were used to control the registration process. Since we are dealing with the beating heart and in many situations with gated MPI acquisitions, this may lead to inaccurate results. Woo and coworkers ${ }^{[6]]}$ developed a registration method to account for phase mismatch between CTA and SPECT. A fully automated segmentation of LV myocardium and blood pool was performed for all gated datasets and new masked volumetric datasets created. All new MPI volumes were rigidly registered to the CT volume after Gaussian smoothing. This process is followed by an energy functional with a piecewise constant image model for the three segmented regions, (myocardium, blood pool and extra cardiac structures) was constructed and minimized by means of gradient descent algorithm using a region-based SSD as similarity measure. The frame that best matched the 


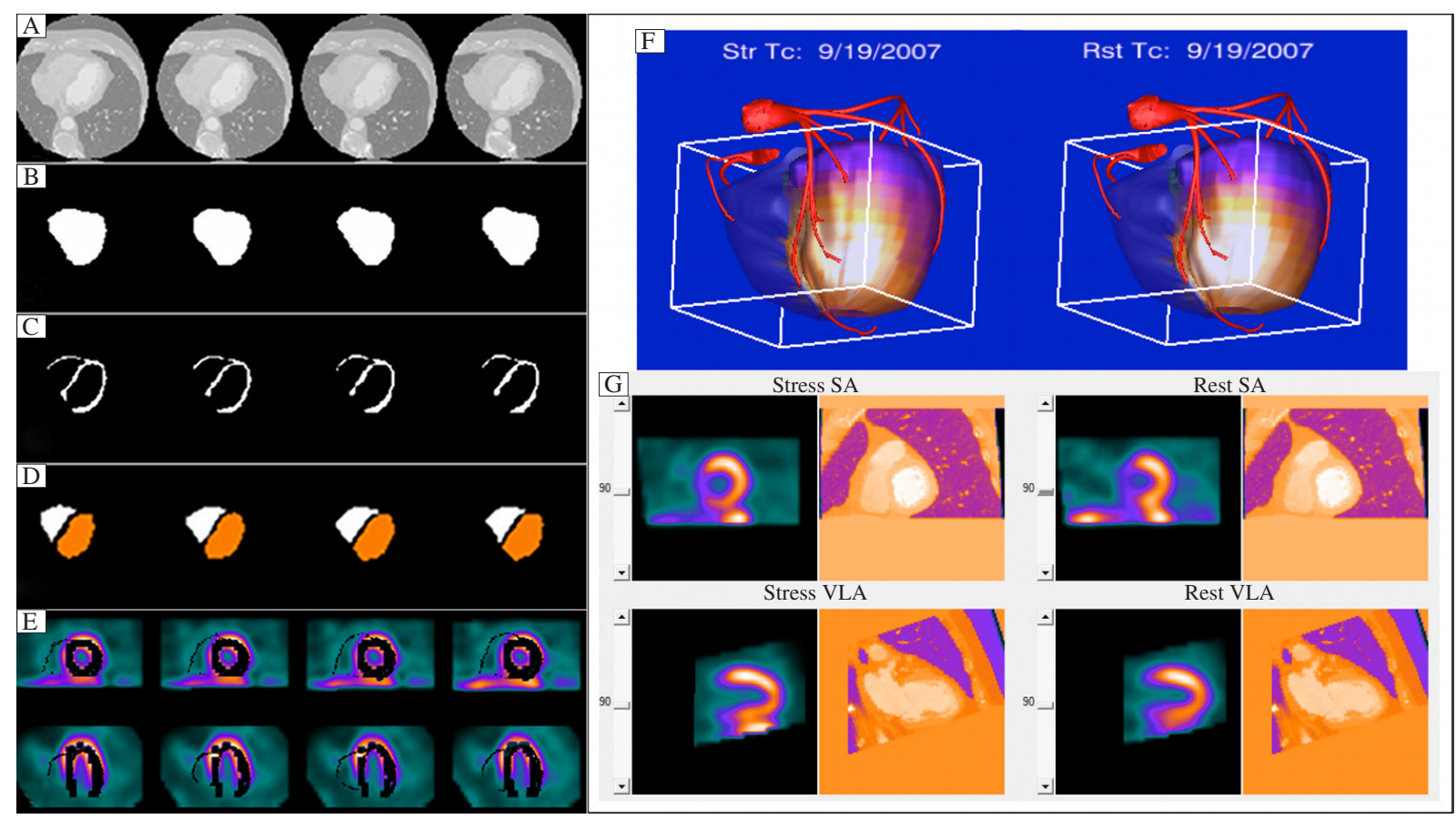

Fig. 4 One case example of image fusion based on Faber et al ${ }^{[70]}$ performed with $\mathbf{E C T b}^{[23]}$. Left - preliminary image processing steps for extraction of anatomical data from CTA: selected slices of original CTA images (A), heart shape (B), myocardium (C), left and right chambers (D); one iteration of the image registration procedure based on mutual information (E). Right - Fused displays. F: 3D surface rendering of RV, LV and coronary trees extracted from the CTA; RV surface is opaque, LV surface has mapped perfusion values from stress/rest SPECT; G: short and horizontal axis slices of synchronized CTA and SPECT scans.

$\mathrm{CT}$ was identified and all the remaining frames were warped by means of thin-plate spline algorithms and summed to create a better static image with less motion artifact. This was finally fused with the CTA and the CTA-derived coronary anatomy.

Image registration algorithms should take advantage of all the available information present in the input images. Given the characteristics of the MPI images, the majority of algorithms employs only the LV position and orientation to guide the registration process. In an article by Faber et al. ${ }^{[70]}$ the right ventricle (RV) was also included in a voxel-based registration algorithm to fuse CTA and SPECT acquisitions. Since multimodality image registration is particularly challenging due to the high differences in image intensities and features, in this work, pre-processing steps were applied to the CTA volume to make it look more similar to the MPI so that mutual information could be used to guide more reliably the alignment. CTA images were manually segmented by means of an in-house software program: endocardial and epicardial borders for both LV and RV delineated. The LV chamber was automatically identified and reoriented into short axis slices for both CTA and SPECT ${ }^{[23]}$. The two LV volumetric masks were rigidly registered by maximizing their product over all possible 3D rotations and translations. This initial transformation was then applied to the full CTA binary mask, smoothed with a Gaussian filter and multiplied to the original MPI image to remove all SPECT extra cardiac activity. The final registration step maximized mutual information between masked MPI and smoothed CTA binary images and mapped the SPECT image to the CTA short axis space; the presence of both RV and LV in the input image should reduce problems of symmetry often encountered when only the LV is used. Should CTAderived coronary anatomy be available, it would be directly fused on the MPI images and surfaces. Fig. 4 shows one case example of image fusion obtained with this methodology.

As more information is included into the modeling, such as anatomy or tissue deformation, the complexity of the algorithms and numerical techniques required to solve the registration problem increases consistently. While the accuracy of the integration may benefit from the more advanced approaches and their application is greatly desired, the final translation into a clinical environment may be affected in terms of computational requirements and time constraints.

\section{LIMITATIONS}

Although significant amount of work has been carried out in the specific field of multimodality image 
registration for $\mathrm{CAD}$ assessment and its additional diagnostic value is widely accepted and demonstrated, image fusion techniques have not completely been translated into clinical routine. The main reason resides in the fact that important parts of the registration techniques proposed and even successfully validated still require intensive manual interaction. This is particularly true for the anatomical information extraction. Differently from nuclear cardiology that has witnessed and benefited from the development of robust software tools for image quantification, automatic segmentation of the coronary artery tree, the myocardium and even the blood pools from anatomical acquisitions are far from established. Additional work will be needed in the image processing field to allow a straightforward and seamless inclusion of image fusion into the routinely used clinical tools.

Hybrid PET/CT and SPECT/CT scanners are considered promising solutions to these issues since both functional and anatomical datasets are acquired at the same moment and within the same device no registration is in theory needed. In practice, respiratory and heart motion artifacts will still be present and require software approaches to be controlled and minimized. Additionally, while allowing immediate image fusion and direct visualization, hybrid systems will lack the strength of quantitative evaluations that greatly increase image fusion potentiality to solve borderline cases.

A crucial limitation for the multimodality fusion of stand-alone acquisitions is the cumulative radiation dose the patient would receive with the sequential imaging. Although this is still under debate, new devices and new protocols in both the computed tomography and nuclear cardiology fields are continuously being tested with the final goal of reducing radiation while still maintaining efficiency and accuracy.

\section{CONCLUSIONS}

Clinical evidence suggests that image fusion can be a reliable and useful tool in the hands of clinicians for a more accurate diagnosis of $\mathrm{CAD}$, specifically for ambiguous and borderline cases. Additional technical developments in the extraction of anatomical information are still in order for the whole procedure to become fully automated and enter the clinical practice.

\section{References}

[1] Go AS, Mozaffarian D, Roger VL, Benjamin EJ, Berry JD, Borden WB, et al. Heart Disease and Stroke Statistics - 2013 Update: A Report From the American Heart Association. Circulation 2013; 127: e6-e245.

[2] Roger VL, Go AS, Lloyd-Jones DM, Adams RJ, Berry JD, Brown TM, et al. Heart Disease and Stroke Statistics
- 2011 Update: A report From the American Heart Association. Circulation 2011; 123: el8-e209.

[3] Heidenreich PA, Trogdon JG, Khavjou OA, Butler J, Dracup K, Ezekowitz MD, et al. Forecasting the Future of Cardiovascular Disease in the United States: a policy statement from the American Heart Association. Circulation 2011; 123: 933-44.

[4] Kramer CM. Integrated approach to ischemic heart disease. The one-stop shop. Cardiac Magnetic Resonance Imag 1998; 16: 267-76.

[5] Santana CA, Garcia EV, Faber TL, Sirineni GKR, Esteves FP, Sanyal R, et al. Diagnostic performance of fusion of myocardial perfusion imaging (MPI) and computed tomography coronary angiography. J Nucl Cardiol 2009; 16: 201-11.

[6] Budoff MJ, Dowe D, Jollis JG, Gitter M, Sutherland J, Halamert E, et al. Diagnostic performance of 64-multidetector row coronary computed tomographic angiography for evaluation of coronary artery stenosis in individuals without known coronary artery disease: results from the prospective multicenter ACCURACY (Assessment by Coronary Computed Tomographic Angiography of Individuals Undergoing Invasive Coronary Angiography) trial. J Am Coll Cardiol 2008; 52: 172432.

[7] Meijboom WB, Meijs MF, Schuijf JD, Cramer MJ, Mollet NR, van Mieghem CA, et al. Diagnostic accuracy of 64-slice computed tomography coronary angiography: a prospective, multicenter, multivendor study. J Am Coll Cardiol 2008; 52: 2135-44.

[8] Miller JM, Rochitte CE, Dewey M, Arbab-Zadeh A, Niinuma H, Gottlieb I, et al. Diagnostic performance of coronary angiography by 64-row CT. N Engl J Med 2008; 359:2324-36.

[9] Di Carli MF, Hachamovitch R. New technology for noninvasive evaluation of coronary artery disease. Circulation 2007; 115: 1464-80.

[10] Blankstein R, Di Carli MF. Integration of coronary anatomy and myocardial perfusion imaging. Nat Rev Cardiol 2010; 7: 226-36.

[11] Beller GA. Recent advances and future trends in multimodality cardiac imaging. Heart Lung Circ 2010; 19: 193-209.

[12] Achenbach S, Friedrich MG, Nagel E, Kramer CM, Kaufmann PA, Farkhooy A, et al. CV Imaging: what's new in 2012? J Am Coll Cardiol Img 2013; 6: 714-34.

[13] Litt HI, Gatsonis C, Snyder B, Singh H, Miller CD, Entrikin DW, et al. CT Angiography for safe discharge of patients with possible acute coronary syndromes. $N$ Engl J Med 2012; 366: 1393-403.

[14] Gaemperli O, Schepis T, Valenta I, Koepli P, Husmann L, Scheffel H, et al. Functionally relevant coronary artery disease: comparison of 64-section CT angiography with myocardial perfusion SPECT. Radiology 2008; 248 : 414-23.

[15] Hacker M, Jakobs T, Hack N, Nikolaou K, Becker C, von Ziegler F, et al. Sixty-four slice spiral CT angiogra- 
phy does not predict the functional relevance of coronary artery stenoses in patients with stable angina. Eur J Nucl Med Mol Imaging 2007; 34: 4-10.

[16] Rispler S, Keidar Z, Ghersin E, Roguin A, Soil A, Dragu $\mathrm{R}$, et al. Integrated single-photon emission computed tomography and computed tomography coronary angiography for the assessment of hemodynamically significant coronary artery lesions. J Am Coll Cardiol 2007; 49: 1059-67.

[17] Di Carli MF, Dorbala S, Curillova Z, Kwong RJ, Goldhaber SZ, Rybicki FJ, et al. Relationship between CT coronary angiography and stress perfusion imaging in patients with suspected ischemic heart disease assessed by integrated PET-CT imaging. J Nucl Cardiol 2007; 14; 799-809.

[18] Hachamovitch R, Hayes SW, Friedman JD, Cohen I, Berman DS. Comparison of the short-term survival benefit associated with revascularization compared with medical therapy in patients with no prior coronary artery disease undergoing stress myocardial perfusion single photon emission computed tomography. Circulation 2003, 107: 2900-7.

[19] Min JK, Berman DS, Dunning A, Achenbach S, AlMallah M, Budoff MJ, et al. All-cause mortality benefit of coronary revascularization vs. medical therapy in patients without known coronary artery disease undergoing coronary computed tomographic angiography: results from CONFIRM (COronary CT Angiography EvaluatioN For Clinical Outcomes: An InteRnational Multicenter Registry). Eur Heart J 2012; 33: 3088-97.

[20] McCullough CH, Primak AN, Braun N, Koefler J, Yu L, Christner J. Strategies for reducing radiation dose in CT. Radiol Clin North Am 2009; 47: 27-40.

[21] Garcia EV. Quantitative nuclear cardiology: we are almost there! J Nucl Cardiol 2012; 19: 424-37.

[22] Klocke FJ et al. ACC/AHA/ASNC guidelines for the clinical use of cardiac radionuclide imaging-executive summary: a report of the American College of Cardiology/American Heart Association Task Force on Practice Guidelines (ACC/AHA/ASNC committee to revise the 1995 guidelines for the clinical use of cardiac radionuclide imaging). Circulation 2003; 108: 1404-18.

[23] Garcia EV, Faber TL, Cooke CD, Folks RD, Chen J, Santana C. The increasing role of quantification in nuclear cardiology: The Emory approach. $J$ Nucl Cardiol 2007; 14: 420-32.

[24] Germano G, Kavanagh PB, Slomka PJ, Van Kriekinge SD, Pollard G, Berman DS. Quantitation in gated perfusion SPECT imaging: The Cedars-Sinai approach. $J$ Nucl Cardiol 2007; 14: 433-54.

[25] Ficaro EP, Lee BC, Kritzman JN, Corbett JR. Corridor4DM: The Michigan method for quantitative nuclear cardiology. J Nucl Cardiol 2007; 14: 455-65.

[26] Underwood SR et al. Myocardial perfusion scintigraphy: the evidence. Eur J Nucl Med Mol Imaging 2004; 31: 261-91.

[27] Berman DS, Hachamovitch R. Risk assessment in patients with stable coronary artery disease: incremental value of nuclear imaging. J Nucl Cardiol 1996, 3(S): 41-9.

[28] Hachamovitch R, Di Carli MF. Methods and limitations of assessing new noninvasive tests. Part II: outcomesbased validation and reliability assessment of noninvasive testing. Circulation 2008; 117: 2793-801.

[29] Hachamovitch R, Hayes SW, Friedman JD, Cohen I, Berman DS. A prognostic score for prediction of cardiac mortality risk after adenosine stress myocardial perfusion scintigraphy. J Am Coll Cardiol 2005; 45: 722-9.

[30] Hachamovitch R, Hayes S, Friedman JD, Cohen I, Shaw LJ, Germano G, Berman DS. Determinants of risk and its temporal variation in patients with normal stress myocardial perfusion scans: what is the warranty period of a normal scan? J Am Coll Cardiol 2003; 41: 1329-40.

[31] Cerqueira MD, Weissman NJ, Dilsizian V, et al. Standardized myocardial segmentation and nomenclature for tomographic imaging of the heart: a statement for healthcare professionals from the Cardiac Imaging Committee of the Council on Clinical Cardiology of the American Heart Association. Circulation 2002; 105: 539-42.

[32] Kalbfleisch H, Hort W. Quantitative study on the size of coronary artery supplying areas postmortem. Am Heart J 1977; 183-8.

[33] Sheth T, Yusuf S. Enhancing risk prediction with PET coronary flow reserve. J Am Coll Cardiol Img 2012; 5: 1035-6.

[34] Fiechter M, Ghadri JR, Gebhard C, Fuchs TA, Pazhenkottil AP, Nkoulou RN, Herzog BA, Wyss CA, Gaemperli O, Kaufmann PA. Diagnostic value of $13 \mathrm{~N}$ ammonia myocardial perfusion PET: added value of myocardial flow reserve. J Nucl Med 2012; 53:1230-4.

[35] Murthy VL, Naya M, Foster CR, et al. Association between coronary vascular dysfunction and cardiac mortality in patients with and without diabetes mellitus. Circulation 2012; 126: 1858-68.

[36] Gaemperli O, Husmann L, Schepis T, Koepfli P, Valenta I, Jenni W, et al. Coronary CT angiography and myocardial perfusion imaging to detect flow-limiting stenoses: a potential gatekeeper for coronary revascularization? Eur Heart J 2009; 30: 2921-9.

[37] Rispler S, Keidar Z, Ghersin E, Roguin A, Soil A, Dragu $\mathrm{R}$, et al. Integrated single-photon emission computed tomography and computed tomography coronary angiography for the assessment of hemodynamically significant coronary artery lesions. J Am Coll Cardiol 2007; 49: 1059-67.

[38] Garcia EV, Gropler RJ. Eighth nuclear cardiology invitational conference Annapolis, Maryland, 2008. J Nucl Cardiol 2008; 15(6): e37-50.

[39] Gaemperli O, Schepis T, Valenta I, Husmann L, Scheffel $\mathrm{H}$, Duerst V, et al. Cardiac image fusion fro stand-alone SPECT and CT: clinical experience. J Nucl Med 2007; 48: 696-703.

[40] Smolka PJ, Baum RP. Multimodality image registration with software: state of the art. Eur J Nucl Med Mol Im- 
aging 2009; 1(S): 44-55.

[41] Slomka PJ. Software approach to merging molecular with anatomic information. $J$ Nucl Med 2004; 45(S): 3645.

[42] BF Hutton, Braun M, Thurfjell L, Lau DYH. Image registration: an essential tool for nuclear medicine. Eur $J$ Nucl Med 2002; 29: 559-77.

[43] Maintz JB, Viergenev MA. A survey of medical image registration. Med Image Anal 1998; 2: 1-36.

[44] Hill DLG, Batchelor PG, Holden M, Hawkes DJ. Medical image registration. Phys Med Biol 2001; 46:R1-R45.

[45] Beg MF, Miller MI, Younes ATL. Computing metrics via geodesics on flows of diffeo-morphisms. Int J Comput Vis 2005; 61: 139-57.

[46] Thirion JP. Image matching as a diffusion process: An analogy with Maxwell's demons. Med Image Anal 1998; 2: 243-60.

[47] R Bajcsy, S Kovacic. Multiresolution elastic matching. Comput Vis Graph Image Process 1989; 46: 1-21.

[48] D Rueckert, P Aljabar. Nonrigid registration of medical images: theory, methods and applications. IEEE Signal Process Mag 2010; 7: 113-9.

[49] Audette MA, Ferrie FP, Peters TM. An algorithmic overview of surface registration techniques for medical imaging. Med Image Anal 2000; 4: 201-217.

[50] Besl PJ, McKay ND. A method for registration of 3D shapes. IEEE Trans PAMI 1992; 14: 239-56.

[51] Pelizzari CA, Chen GTY, Spelbring DR, Weichselbaum RR, Chen CT. Accurate three-dimensional registration of CT, PET and/or MR images of the brain. J Comput Assist Tomogr 1989; 13: 20-6.

[52] Faber TL, McColl RW, Opperman RM, Corbett JR, Peshock RM. Spatial and temporal registration of cardiac SPECT and MR images: methods and evaluation. Radiology 1991; 179: 857-61

[53] Scott AM, Macapinlac HA, Divgi CR, Zhang JJ, Kalaigian $\mathrm{H}$, Pentlow $\mathrm{K}$, et al. Clinical validation of SPECT and CT/MRI image registration in radiolabeled monoclonal antibody studies of colorectal carcinoma. $\mathrm{J} \mathrm{Nucl}$ Med 1994; 35: 1976-84.

[54] Shannon CE. The mathematical theory of communication (Part I and II). Bell Syst Tech J 1948; 27: 379-423, 623-56.

[55] Wells WM, Viola P, Atsumi H, Nakajima S, Kikinis R. Multi-modal volume registration by maximization of mutual information. Med Image Anal 1996; 1: 35-51.

[56] Studholme C, Hill DL, Hawkes DJ. Automated 3-D registration of MR and CT images of the head. Med Image Anal 1996; 1: 163-175.

[57] Maes F, Collignon A, Vandermeulen D, Marchal G, Suetens P. Multimodality image registration by maximization of mutual information. IEEE Trans Med Imaging 1997; 16: 187-98.

[58] Pluim JP, Maintz JB, Viergever MA. Mutual-information-based registration of medical images: a survey. IEEE Trans Med Imaging 2003; 22: 986-1004.
[59] Press WH, Teukolsky SA, Vetterling WT, Flannery BP. Numerical recipes in $\mathrm{C}++$, 3nd edn. Cambridge: Cambridge University Press, 2007.

[60] Peifer JW, Garcia EV, Cooke CD, Klein JL, Folks R, Ezquerra NF. 3-D Registration and Visualization of Reconstructed Coronary Arterial Trees on Myocardial Perfusion Distributions. Visualization in Biomedical Computing, Richard A Robb Editor Proc, SPIE, 1992, 1808, 225-34.

[61] Faber TL, Klein JL, Folks RD, Hoff JG, Euhevg JW, Malveaux EJ, et al. Automatic Unification of ThreeDimensional Cardiac Perfusion with Three-dimensional Coronary Artery Anatomy. Computers in Cardiology 1996; 0276-6547: 333-6

[62] Garcia EV, Cooke CD, Van Train KF, Folks R, Peifer J, DePuey EG, et al. Technical aspects of myocardial SPECT imaging with technetium-99m sestamibi. Am J Cardiol 1990;190: 23E-31E.

[63] Peifer JW, Ezquerra NF, Cooke CD, Mullick R, Klein L, Hyche ME, et al. Visualization of multimodality cardiac imagery. IEEE Trans Biomed Eng 1990; 37: 744-56.

[64] Schindler TH, Magosaki N, Jeserich M, Oser U, Krause T, Fischer R, et al. Fusion imaging: combined visualization of $3 \mathrm{D}$ reconstructed coronary artery tree and $3 \mathrm{D}$ myocardial scintigraphic image in coronary artery disease. Int J Card Imaging 1999; 15: 357-68.

[65] Faber TL, Santana CA, Garcia EV, Candell-Riera J, Folks RD, Peifer JW, et al. Three-dimensional fusion of coronary arteries with myocardial perfusion distribution: clinical validation. J Nucl Med 2004; 45: 745-53.

[66] Faber TL, Chiron F, Ezquerra NF, et al. Registration of multimodal $3 \mathrm{D}$ cardiac information using the iterative closest point approach. In: Wilson DC, Tagare HD, Bookstein FL, et al., eds. Mathematical Modeling, Estimation, and Imaging. Proceedings SPIE 4121. Bellingham, WA: SPIE; 2000: 233-41.

[67] Gaemperli O, Schepis T, Kalff V, Namdar M, Valenta I, Stefani L, et al. Validation of a new cardiac image fusion software for three-dimensional integration of myocardial perfusion SPECT and stand-alone 64-slice CT angiography. Eur J Nucl Med Mol Imaging 2007; 34: 1097-106.

[68] Slomka PJ, Cheng VY, Dey D, Woo J, Ramesh A, van Kriekinge S, et al. Quantitative analysis of myocardial perfusion SPECT anatomically guided by coregistered 64-slice coronary CT angiography. J Nucl Med 2009; 50: $1621-30$

[69] Woo J, Slomka PJ, Dey D, Cheng VY, Hong BW, Ramesh A, et al. Geometric feature-based multimodal image registration of contrast-enhanced cardiac CT with gated myocardial perfusion SPECT. Med Phys 2009; 36: 5467-79.

[70] Faber TL, Santana CA, Piccinelli M, Nye JA, Votaw JR, Garcia EV, et al. Automatic alignment of myocardial perfusion images with contrast-enhanced cardiac computed tomography. IEEE Trans Nuclear Science 2011; 58: 2296-302. 